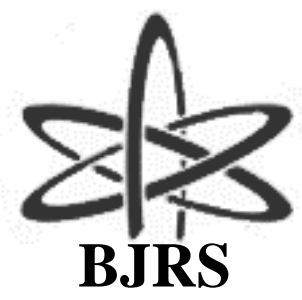

\author{
BRAZILIAN JOURNAL \\ $\mathrm{OF}$ \\ RADIATION SCIENCES \\ 08-03A (2020) 01-12
}

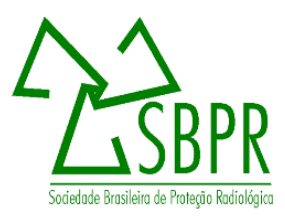

\title{
New 3D Diffusion Code Based on The Nodal Polynomial Expansion
}

\author{
Jadna Mara Santos Mendes, Sérgio Barros Paixão, Sergio de Oliveira Vellozo \\ Universidade Federal do Rio de Janeiro \\ jadnamsm@gmail.com
}

\begin{abstract}
Nodal Expansion Method (NEM) is widely employed in the neutronic design of nuclear reactors core. The main reason is its higher computational performance and efficiency when compared with the conventional fine mesh difference method or finite element method. The NEM diffusion calculation uses coarse spatial meshes and the size can lie in assembly scale. This is the key for the computational efficiency and high accuracy calculations. The NEM consists mainly of basis polynomial function expansion for each nodal direction. The Nodal Expansion Method (NEM), as proposed by Finnemann in 1977, has been used to solve the multigroup neutron diffusion equations in three-dimensional (3D) rectangular geometry. The weighted residual technique has been applied to determine the higher order coupling coefficients. Resulting from the combination of nodal and finite element methods, NEM provides rigorously accurate equations obtained by integrating the neutron balance equation. The two group coarse mesh 3D IAEA benchmark has been simulated by NEM3D-1A using different nodes sizes. The VENTURE keff result was taken as reference. The NEM3D-1A calculations were found to be in good agreement with those obtained by using other standard codes and our $\Delta$ keff deviation from VENTURE result become less than $0.002 \%$.
\end{abstract}

Keywords: Diffusion, Nodal, Polynomial, LWR, Coarse Mesh. 


\section{INTRODUCTION}

Efficient and safe operation of a nuclear power plant (NPP) represents a challenge to nuclear reactor design and operation engineers. The prediction of the behavior and performance of a nuclear reactor is essential to this task.

Several characteristics of the reactor must be established to ensure that the reactor can be started up, operated at a steady state over its entire operating lifetime, and shut down as needed in a safe and economic manner. Some of the parameters and phenomena, which must be predicted with sufficient accuracy, are the spatial distribution of the power generation throughout the operational period, the positioning of control rods within the core as required for control of the nuclear reactor, and the time-dependent behavior of the spatial power distribution during off-normal and accident situations.

The steady-state core analysis of both new reactor designs and the reload cores of operating reactors involves a large number of whole core calculations in order to optimize loading patterns and determine reload safety parameters.

In result, even at the current speed of computational machinery, it is absolutely impractical to perform all of these calculations by applying fine mesh methods to a model containing detail at the level of individual fuel rods, control elements, and coolant regions in an entire reactor core [1]. For this reason, nodal methods are currently widely used to predict neutron flux behavior of a reactor core.

The efficiency of nodal methods is very high and comparable with the finite difference methods [2]. It is very fast and allows us to compute such huge number of reactor states. It must be added, using this method one can get only approximate information about the neutron flux in a single node (or coarse-mesh area, usually a single fuel assembly transversal dimension). This analysis requires many subsequent calculations of the flux and power distributions for the fuel assemblies while there is no need for detailed distribution within the assembly. For obtaining detailed distribution within the assembly the heterogeneous flux reconstruction must be applied [3]. This work is organized as follow: the section 2 shows the so called Basic Nodal Method with flux quadratic polynomial 
expansion and superior order expansion and the IAEA-3D benchmark used as reference; the section 3 contains the results and in section 4 contains the conclusion and some remarks.

\section{NODAL POLYNOMIAL EXPANSION METHOD}

The Nodal Expansion Method consists of a technique conceptually derived from a class of Nodal Methods (NM), to which were combined some characteristics of the finite element method [4]. The NEM, as will be presented in this work [5], will have its formulation done through diffusion equations set for the mean flux, for each spatial direction. The nodal equations are obtained through the formal integration of the diffusion equations in each control volume called node.

$$
\begin{gathered}
\sum_{u=x, y, z} \frac{1}{a_{u}^{m}}\left\{\left(J_{g u r}^{+m}-J_{g u r}^{-m}\right)-\left(J_{g u l}^{+m}-J_{g u l}^{-m}\right)\right\}+\Sigma_{t g}^{m} \Phi_{g}^{m}= \\
=\sum_{g^{\prime}=1}^{G}\left\{\Sigma_{g g^{\prime}}^{m}+\frac{1}{\lambda} \chi_{g}^{m} v \Sigma_{f g^{\prime}}^{m}\right\} \Phi_{g}^{m}
\end{gathered}
$$

$\Phi_{g}^{m}=\frac{1}{V_{m}} \int_{V_{m}} \phi_{g}(x, y, z) d V$ is the nodal mean flux of energy group $\mathrm{g}$.

$\Sigma_{t g}^{m}$ is the total macroscopic cross section of energy group $g$ and node $m$.

$\Sigma_{g g^{\prime}}^{m}$ is the transfer macroscopic cross section from energy group $g^{\prime}$ to energy group $g$.

$\chi_{g}^{m}$ is the normalized fission spectra for energy group $g$ and node $m$.

$v \Sigma_{f g}^{m}$ is the mean neutron released by fission multiplied by the fission macroscopic cross section for energy group $g$ and node $m$.

$\lambda$ is the multiplication factor eigenvalue.

$a_{u}^{m}$ is the node ${ }^{m}$ size and $u$ direction.

$I_{g u r}^{+m}, I_{g u r}^{-m}, I_{g u l}^{+m}$ and $I_{g u l}^{-m}$ are, respectively, partial neutron currents of node $m$, at right and left wall, energy group $g$ and direction $u$.

Figures 1 and 2 shows how the mean nodal flux and partial currents, in one direction $u$, are distributed by the node. 
Figure 1: Partial currents at node $\boldsymbol{m}$ and the mean nodal flux $\boldsymbol{\Phi}_{g}^{m}$.

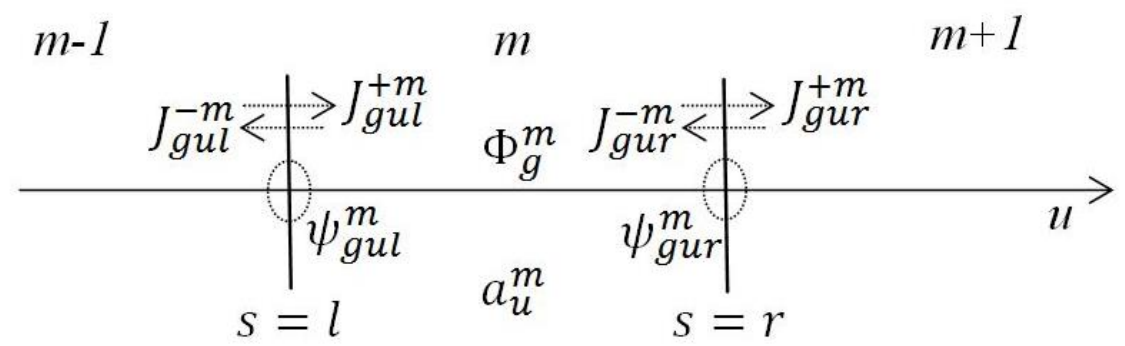

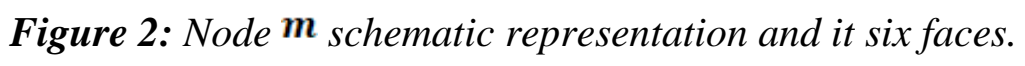
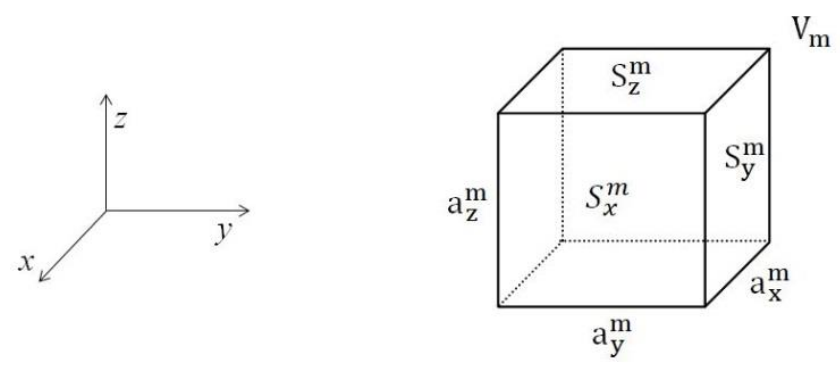

The eq. (1) is a perfect neutron balance within node $\mathrm{m}$. The partial currents, responsible for the couplings between the nodes, will be determined through the use of the Fick's Law. The onedimensional flux will be represented by a polynomial. The coefficients will be determined by the weighted residuals techniques, allowing nodes of $20 \mathrm{~cm}$ in length.

\subsection{Second order expansion - Basic NEM}

The polynomial expansion method consists in write and approximates the one dimensional flux as a sum of polynomials base functions as:

$$
\psi_{g u}^{m}(u) \cong C_{00, g}^{m} h_{0}\left(\frac{u}{a_{u}^{m}}\right)+\sum_{k=1}^{N} C_{u k, g}^{m} h_{k}\left(\frac{u}{a_{u}^{m}}\right)
$$


Where $C_{u k, g}^{m}$ is the coupling coefficient of $k$ order, $C_{00, g}^{m}$ is the fundamental coefficient and $N$ is the approximation order. For the current case, $N=2$ and the expansion becomes:

$$
\psi_{g u}^{m}(u) \cong C_{00, g}^{m} h_{0}\left(\frac{u}{a_{u k}^{m}}\right)+C_{u 1, g}^{m} h_{1}\left(\frac{u}{a_{u}^{m}}\right)+C_{u 2, g}^{m} h_{2}\left(\frac{u}{a_{u k}^{m}}\right)
$$

Equations for partial currents can be obtained by the substitution of eq. (3) in balance eq. (1) evaluated in node surfaces. The base functions are:

$$
\left\{\begin{array}{c}
h_{0}(x)=1 \\
h_{1}(x)=2 x-1 \\
h_{2}(x)=6 x(1-x)-1
\end{array} \quad ; \quad x=\frac{u}{a_{u}^{m}}\right.
$$

This expansion is totally consistent with all interface conditions. This means that the number of constants $C_{u k, g}^{m}$ matches exactly the number of interfaces and boundary conditions.

\subsection{IAEA-3D Numerical Benchmark}

The IAEA-3D Benchmark [6] consists of a commercial PWR reactor (177 assemblies and octant symmetry). The core dimensions are $170 \mathrm{~cm}$ in radius and $380 \mathrm{~cm}$ in height. The assembly pitch is $20 \mathrm{~cm}$. There are five distinct regions. Figures 3,4 and 5 show a schematic 3D view, radial cross section and axial cross section, respectively. Table 1 presents the macroscopic cross sections for each region of the simulated core. 
Figure 3: 3D IAEA PWR core.

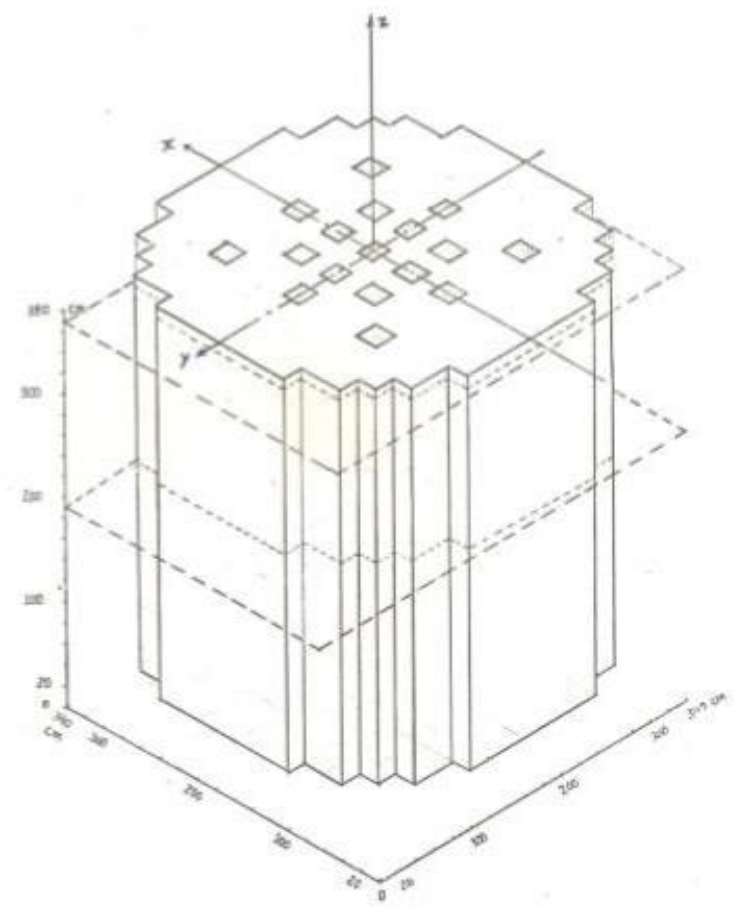

Figure 4: The core transversal cross section.

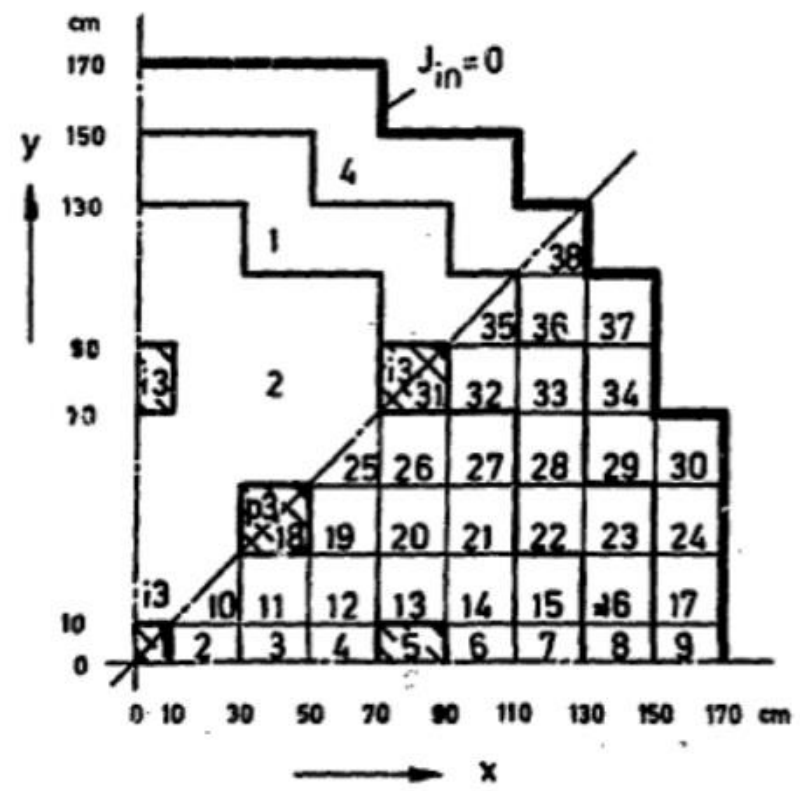

Source: ANL-7416, 1977 
Figure 5: The core longitudinal cross section with control rods.

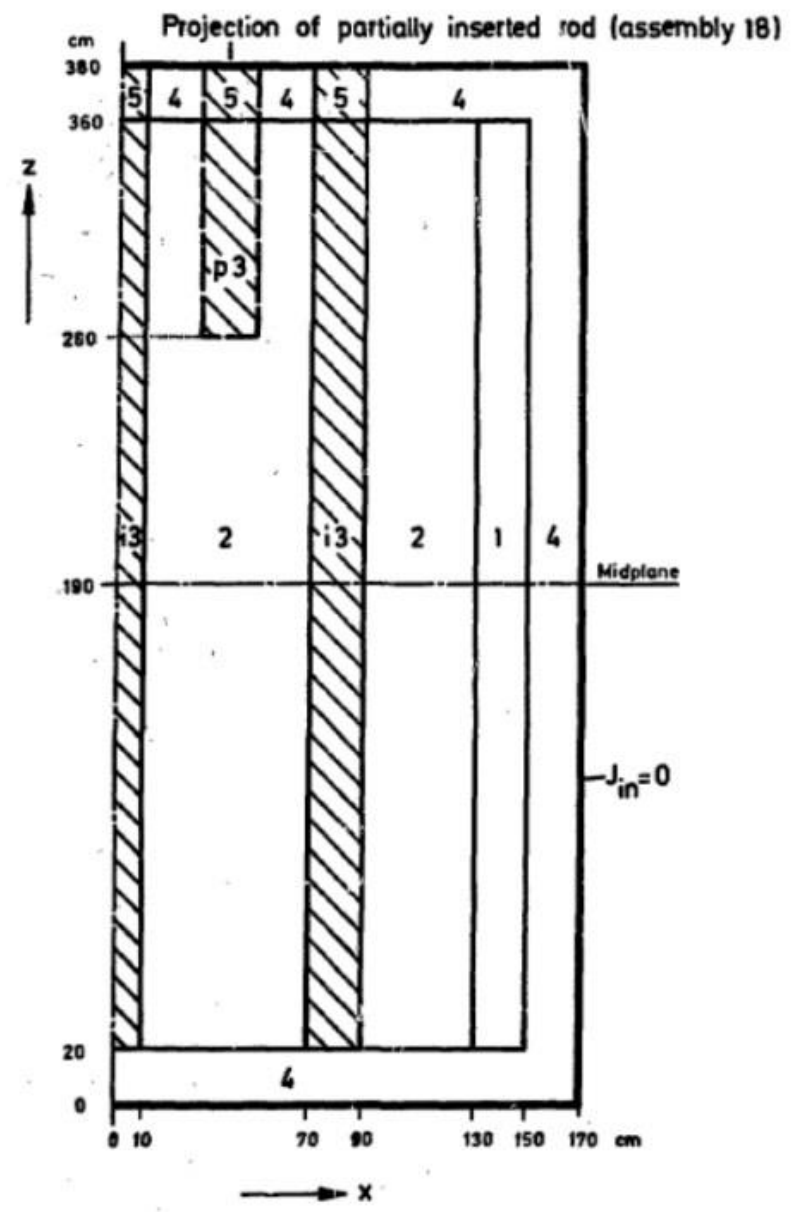

Source: ANL-7416, 1977

Table 1: Nuclear data for IAEA benchmark (ANL-7416, 1977).

\begin{tabular}{cccccccc}
\hline Region & $D_{1}$ & $D_{2}$ & $\Sigma_{1 \rightarrow 2}$ & $\Sigma_{a 1}$ & $\Sigma_{a 2}$ & $v \Sigma_{f 2}$ & Material \\
\hline 1 & 1.5 & 0.4 & 0.02 & 0.01 & 0.08 & 0.035 & Fuel 1 \\
2 & 1.5 & 0.4 & 0.02 & 0.01 & 0.085 & 0.035 & Fuel 2 \\
3 & 1.5 & 0.4 & 0.02 & 0.01 & 0.13 & 0.035 & Fuel 2+Rod \\
4 & 2.0 & 0.3 & 0.04 & 0 & 0.01 & 0 & Reflector \\
5 & 2.0 & 0.3 & 0.04 & 0 & 0.055 & 0 & Refl.+Rod \\
\hline
\end{tabular}


The eigenvalue and mean radial power distribution calculated by VENTURE using extrapolated mesh spacing technique are taken as the reference (benchmark) results [7]. Its mean radial power distribution in octant symmetry is shown in Figure 6.

Figure 6: The IAEA-3D benchmark mean radial power distribution.

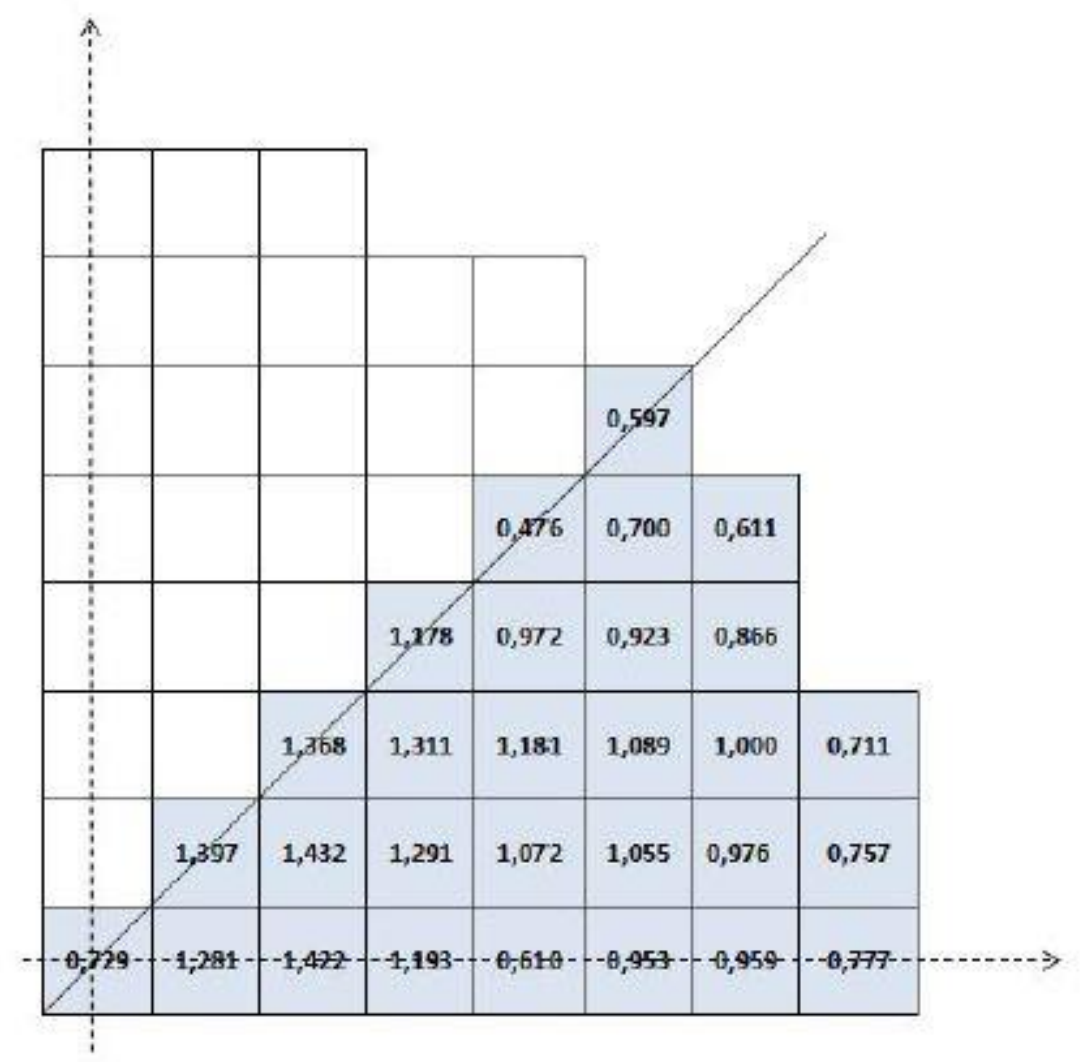

\section{RESULTS}

The $k_{\text {eff }}$ calculated by NEM3D-1A and other standard codes $[7,8,4,9,10,11,12]$ were compared to the reference value (VENTURE using extrapolated mesh spacing technique). The $k_{\text {eff }}$ and associated deviation are compiled in Table 2.

Table 2: The $\boldsymbol{k}_{\text {eff }}$ and deviations from the reference result.

$\begin{array}{llll}\text { CODE } & a_{x} \times a_{y} \times a_{z}(\mathbf{c m 3}) & k_{\text {eff }} & \Delta k_{\text {eff }}(\%)\end{array}$




\begin{tabular}{cccc}
\hline VENTURE & Extrap. & 1.02903 & - \\
VENTURE & $10 \times 10 \times 10$ & 1.02864 & 0.03790 \\
VANCER & $10 \times 10 \times 10$ & 1.02949 & 0.04470 \\
FEM-3D & $10 \times 10 \times 10$ & 1.02920 & 0.01652 \\
IQSBOX & $20 \times 20 \times 20$ & 1.02911 & 0.00777 \\
NEM3D-1A & $20 \times 20 \times 20$ & 1.02901 & 0.00194 \\
Christensen & $20 \times 20 \times 20$ & 1.02896 & 0.00680 \\
ARROTA & $20 \times 20 \times 20$ & 1.02899 & 0.00389 \\
NESTLE & $20 \times 20 \times 20$ & 1.02899 & 0.00389 \\
PARCS & $20 \times 20 \times 20$ & 1.02909 & 0.00641 \\
\hline
\end{tabular}

The Figure 7 shows NEM3D-1A mean radial power distribution deviation in each node of the octant symmetry. The maximum deviation was $0.9986 \%$.

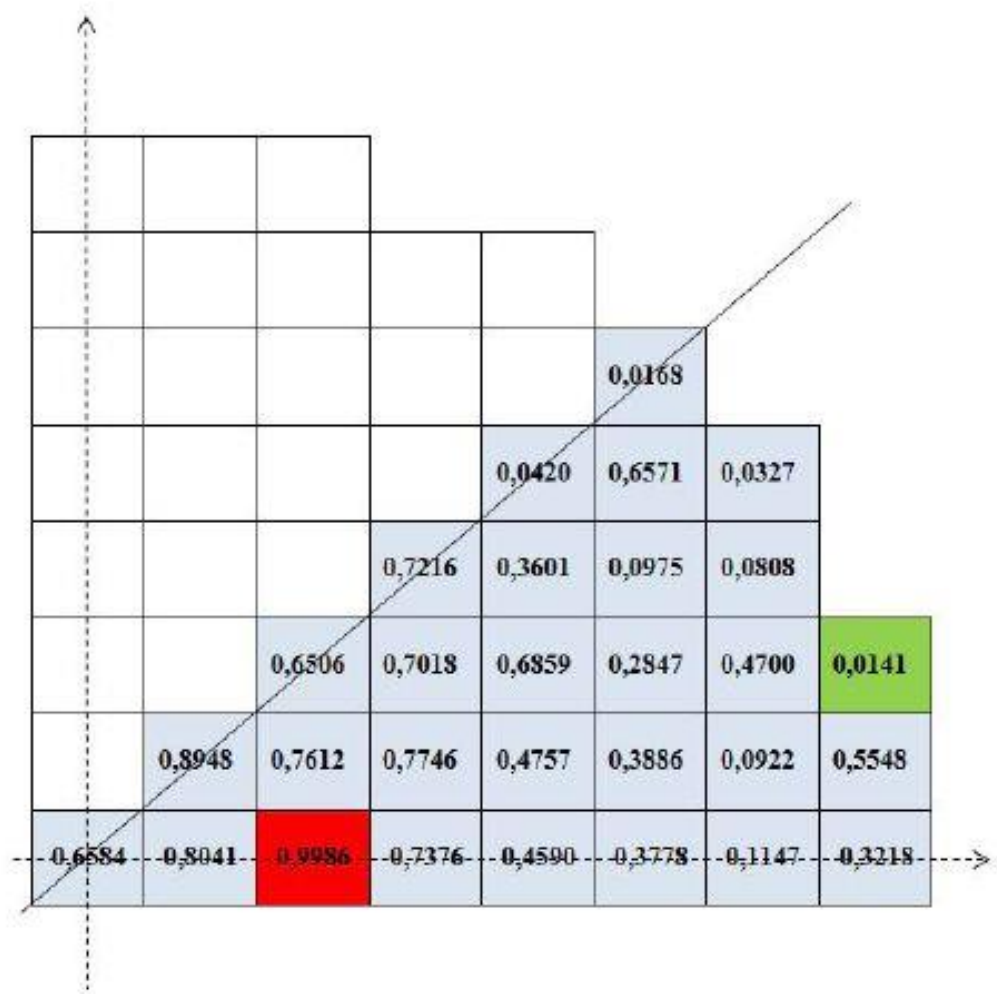


Figure 7: The NEM3D-1A mean radial power distribution deviation.

\section{CONCLUSION}

Quantitatively, NEM3D-1A was able to predict the $k_{\text {eff }}$ with a good accuracy $(0.00194 \%)$, as can be seen in Table 2. NEM3D-1A is also simple and fast. It took less than 2 seconds running in a Dell Optiplex 790 i3 4GB Windows 7 Desktop computer. 


\section{ACKNOWLEDGMENT}

For their support, National Council for the Improvement of Higher Education (CAPES) and National Council for Scientific and Technological Development (CNPq).

\section{REFERENCES}

[1] DORNING, J. J. Modern coarse-mesh methods — a development of the '70's. Proc. Topl. Mtg. Computational Methods of Nuclear Engineering, American Nuclear Society, Williamsburg Virginia, 23-25 April, Vol. 1, pp. 1-3 1979.

[2] “A Consistent Nodal Method for the Analysis of Space-Time Effects in Large LWR's,". 1975. Available at: <https://www.oecd-nea.org/nsd/docs/1975/csni75-5.pdf>. Last accessed: 26 Jun. 2019.

[3] DUDERStadT, J. J., HAMILTON L. J. Nuclear Reactor Analysis, $3^{\text {rd }}$ ed. New York: John Wiley \& Sons, 1976.

[4] "Solution of the multigroup neutron diffusion equations by finite element method,". 1975. Available at: <https://orbit.dtu.dk/files/56550414/RIS_M_1809.pdf〉. Last accessed: 26 Jun. 2019.

[5] FINNEMANN, H., BENNEWITZ, F., WAGNER, M. R. Interface Current Techniques for Multidimensional Reactor Calculations, Atomkenergie, Erlangen Germany, Vol. 30, pp.1231251977.

[6] "Argonne code ccenter: benchmark problem book,". 1977. Available at: <https://www.osti.gov/servlets/purl/5037820>. Last accessed: 26 Jun. 2019.

[7] "VENTURE: A Code Block for Solving Multigroup Neutronics Problems Applying the FiniteDifference Diffusion-Theory Approximation to Neutron Transport,”. 1975. Available at: <https://www.osti.gov/servlets/purl/4158202>. Last accessed: 26 Jun. 2019.

[8] "Test of the Diffusion Theory Difference in Slab Geometry (Computer Code VANCER),". 1978. Available at: <https://www.osti.gov/servlets/purl/6805732>. Last accessed: 26 Jun. 2019. 
[9] “Three-Dimensional Static and Dynamic Reactor Calculations by the Nodal Expansion Method,”. 1985. Available at: <https://orbit.dtu.dk/files/170742623/RISOR496.pdf>. Last accessed: 26 Jun. 2019.

[10] "Static Three-Dimensional ARROTA Benchmarking,". 1985. Available at: <https://www.osti.gov/servlets/purl/527546>. Last accessed: 26 Jun. 2019.

[11] "Validation of NESTLE Against Static Reactor Benchmark Problems,". 1995. Available at: < https://www.osti.gov/servlets/purl/527546> Last accessed: 26 Jun. 2019.

[12] DOWNAR, T. J. et al. PARCS: Purdue Advanced Reactor Core Simulator, International Conference on the New Frontiers of Nuclear Technology: Reactor Physics, Safety and HighPerformance Computing, In: PHYSOR, 2002, Seoul, Korea. 